\title{
WLAN PERFORMANCE IMPROVEMENT BY HEADER COMPRESSION AND PACKET OVERHEARING REDUCTION
}

\author{
David Levy — Ivan Kotuliak — Peter Trúchly *
}

\begin{abstract}
For wireless systems, it is of vital importance to use the scarce radio resources in an efficient way. The main targets are to reduce the power consumption and increase the throughput of wireless devices. Using header compression both throughput can be increased and power can be saved. Using packet overhearing reduction power can be saved. This paper presents the achieved performance improvements when using these two methods. The simulation results are confirmed by analytical calculations. We show that we can achieve up to $35 \%$ power save when combining both methods and close to 0.1 additional normalized throughput.
\end{abstract}

K e y w o r d s: WLAN, 802.11, power consumption, throughput, header compression, packet overhearing

\section{INTRODUCTION}

Currently, WLAN technology is available in a number of various devices, such as laptops, tablets, mobile phones or PDAs. Those devices are becoming increasingly popular due to the increasing presence of wireless LAN hot spots and the use of unlicensed spectrum. In addition to the spectral efficiency and security issues, energy consumption is a vital issue in making the usage of these devices widespread. Efforts are underway in improving power conservation in those devices and thus increasing the duration between recharging the battery. Energy consumption is mainly influenced by the transmission of WLAN frames and their listening and receiving in receivers.

In order to efficiently use these resources, the overhead present in every frame should be kept small. On the other side, most of the layers above the WLAN were developed before the emergence of WLAN and therefore did not consider the wireless resource limitations. The 802.11 MAC layer [1] itself has address fields length which are identical to the wireline 802.3 Ethernet protocol.

This inefficiency is growing with the $802.11 \mathrm{n}$ protocol [2] which requires even more overhead to both support backwards compatibility and new complex optimization algorithms. For example, the 802.11n MAC header contains an additional 4-byte field called HT Control. This field is required for link adaptation control and MIMO calibration.

The header compression relies on difference between consecutive frames. Therefore it increases the risk of forwarding undetected erroneous headers to the upper layers. Existing compression schemes combat this problem by synchronizing the compressor and decompressor with dedicated synchronization frames sent back and forth. Obviously this solution unacceptably increases power consumption and reduces throughput. We propose another method to reduce probability of forwarding erroneous frames to upper layers to a minimum.
In our proposal described in detail in [3], we alleviated mentioned problems and proposed a novel generic compression algorithm.

In [4], we proposed a method to reduce energy consumption of an 802.11 station (STA) in an environment where there is heavy traffic. A standard STA receives complete frames, at full power, before it discards them when it discovers the frames were not addressed to it this is called packet overhearing. We introduced a new PHY state which consumes less power than the active states but can switch back fast to an active state. This new PHY state is used to switch off the receiver immediately after the destination address is received and switch it on towards the assumed end of the frame. Since the receiver energy consumption in this new state is much lower than in reception state, this technique increases battery life of a WLAN device in an environment where the channel is heavily loaded by other STAs.

In [4], we also proposed an analytical model that provides the average power consumption of a standard 802.11 station (STA). The proposed method is based on the Markov analytical model of the 802.11 Distributed Coordinated Function (DCF) proposed by Bianchi in [5] for a network in saturation. The results of the Markov model were developed in [4] to obtain the average power consumption of a STA. We proved that our analytical model is valid for the modeled network.

In this paper we modified the analytical model equations of a standard 802.11 STA to show the performance improvement applying packet overhearing reduction and header compression. We will demonstrate the energy saved compared to a standard STA both analytically and by simulations. This paper continues and extends our investigations done in [6]. We will show that we achieve up to $35 \%$ overall energy consumption reduction in the studied scenarios when combining both methods. We will show that header compression also increases the normalized throughput by almost 0.1 . All results will be analyzed and commented.

\footnotetext{
* Slovak University of Technology, Faculty of Informatics and Information Technologies, Institute of Computer Systems and Networks, Ilkovičova 2, 84216 Bratislava, Slovakia, david.levy@stuba.sk, ivan.kotuliak@stuba.sk peter.truchly@stuba.sk
} 
The organization of the paper is as follow: Section 2 describes the energy consumption issue in WLAN. Section 3 describes existing methods to combat packet overhearing and existing compression methods. Section 4 presents the proposed concept of packet overhearing reduction. Section 5 presents the proposed concept of header compression. Section 6 describes the modifications done on the standard STA analytical model to include the header compression and packet overhearing reduction. Section 7 analyses the packet overhearing reduction improvement in power consumption. Section 8 shows the header compression performance improvements. Section 9 shows the power consumption reduction achieved when combining both methods. Section 10 summarizes the paper and presents future works.

\section{BACKGROUND}

A WLAN PHY can be in several operating states: receive, transmit and sleep states. Typically the sleep states are: doze and idle. In the doze state, the PHY has all its RF circuitry turned off. In this state, the transition to the receive state takes several milliseconds. In idle state, the PHY has all its RF circuitry on and is ready to receive. The only difference with the receive state is that there is no demodulation taking place. A typical 802.11 WLAN device consumes power as follow [6]: $P_{t x}=450 \mathrm{~mW}$ $($ at $15 \mathrm{dBm}), P_{r x}=140 \mathrm{~mW}, P_{\text {idle }}=110 \mathrm{~mW}$ and $P_{\text {doze }}=0.05 \mathrm{~mW}$.

The WLAN MAC controls the state of the PHY. It achieves this by following the Distributed Coordinated Function (DCF) access method, defined in [1]. A STA monitors the channel to determine if another STA is transmitting, before initiating the transmission of a new packet. If the channel is idle for an interval of time that exceeds the distributed interframe space (DIFS), the packet is transmitted. Otherwise, the STA monitors the channel until it is sensed idle for a DIFS interval, when it then generates a random backoff interval chosen in the range $\left[0, C W_{\min }-1\right]$ for an additional deferral time before transmitting. A STA must wait a random backoff time between two consecutive new packet transmissions, even if the medium is sensed idle in the DIFS time. The backoff timer is decremented only when the medium is idle and it is frozen when the medium is sensed busy. After a busy period, the decrementing of the backoff timer resumes only after the medium has been free longer than a DIFS period. A transmission takes place when the timer zeros out. In case of collision, the involved STAs have to double their random backoff interval range, up to a maximum of $m$ times, resulting in a random backoff interval chosen in the range $\left[0,2^{m} C W_{\min }-1\right]$. After a successful transmission, the random backoff interval range is brought back to $\left[0, C W_{\min }-1\right]$. The DCF access method requires the STA to listen to the channel for long periods of time before actual transmission and reception take place. At the end of this listening period, the device shall be capable to immediately switch to reception or transmission state, therefore, the PHY state has to be the idle state. The device can be in power save mode. In this case the PHY can stay in the doze state most of the time and transition to the reception state only for receiving the beacon. A typical WLAN MAC will keep the PHY in receive state when a WLAN frame with a valid PHY header is received. The PHY will be kept in receive state for the whole duration of the packet. Even if the packet was transmitted to another STA, the MAC continues to receive the full packet. The packet is said to be overheard by the WLAN device. In [7], Biswas simulation results show that for high channel load, packet overhearing represents $30 \%$ of all energy expenditure of a STA. This percentage increases with channel load.

\section{PREVIOUS WORKS}

The present art of the existing compression schemes and packet overhearing avoidance methods can be found in [3] and [4] respectively. They will be briefly reminded to the reader here.

\subsection{Existing Compression schemes}

The compression schemes are divided into three types: existing protocols for header compression only, general header compression techniques and a proposal to compress WLAN headers only.

The protocols [8-10] use the redundancy in the difference between consecutive frames to efficiently compress headers. These protocols can only compress a limited number of layers. In case of a new protocol, or even a modification of a supported protocol, the ROHC (for example) has to be redefined, at least for the modified protocol. Any unknown or unsupported protocol is not compressed by ROHC, CRTP or Van Jacobson. This strongly limits the deployment over WLAN which can have many types of applications running above the WLAN MAC layer.

Our proposal, as it is shown in the next sections, relies, similarly to Van Jacobson, on the difference between two consecutive frames. We can then assume that there will be several zeros, meaning no change between consecutive frames. No assumption can be made on the probability of appearance of any other type of difference. Therefore, general compression methods could be used to compress this difference.

The non-adaptive entropy encoding methods, such as the Huffmann encoding [11], have to be ruled out, due to the unknown probabilities of occurrence of any non-zero difference. The adaptive entropy encoding methods, such as the Adaptive Huffmann encoding, require too much processing time.

The field length to compress includes only the headers of a frame. This is typically less than 100 bytes. As described in [12], the dictionary data compression methods (such as Lempel-Ziv-Welch) are inefficient in the first kilobyte of the message.

Amin et al. proposed, in [13], an improvement to the Run-Length Encoding (RLE), called the Bit Stuffed RLE 
to combat the problems of the original RLE compression algorithm. The modifications are: leaving very short runs uncompressed and bit stuffing.

H. S. Kazmi and H. Raja propose in [14] to compress both the WLAN PHY and MAC headers. Each header is compressed differently.

\subsection{Packet overhearing avoidance method}

Biswas proposes, in [7], an efficient way to avoid packet overhearing. The solution relies on the availability of the Clear-To-Send (CTS) packet. The address of the destination node in the CTS packet is checked. If it is not the one of the device, then the PHY is forced to a less consuming power state for the duration appearing in the CTS packet duration field. This corresponds to the duration of the immediately following data packet. The energy gained corresponds to the difference between $P_{r x}$ and the less consuming power state for the duration of the data packet.

In a demand for high bandwidth efficiency, the RequestTo-Send (RTS)/CTS mechanism is unfortunately a step backwards. Even if the CTS and RTS packets are very small, they still have the 802.11 b very long PHY preamble and must be sent at a very low rate to be heard by all stations in the network. In [15], the author compares the throughputs of a $802.11 \mathrm{~g}$ STA when there is a $802.11 \mathrm{~b}$ STA in the BSS and when there is none. In the former, the AP enforces the usage of either the CTS/RTS mechanism or at least the CTS-to-self mechanism. With CTS/RTS mechanism or CTS-to-self mechanism, the $802.11 \mathrm{~g}$ STA throughput is $8 \mathrm{Mbps}$, respectively $13 \mathrm{Mbps}$, while without it is $22 \mathrm{Mbps}$. The old devices that could only support 802.11 b standard already disappeared from the market, replaced by the modern $802.11 \mathrm{~g} / \mathrm{n}$ standard compliant devices. The end user is rightfully convinced to switch to $802.11 \mathrm{~g} / \mathrm{n}$ technologies which are more band efficient and provide a much higher data rate.

Ju found, in [16], that modern devices have their carrier sensing range twice larger than their transmission range. This means that even if a STA cannot validly receive the packet transmitted by a far STA, it can still sense that this far STA is currently transmitting and defer from transmitting. This sharply reduces the existence of the hidden node.

The two problems that the RTS/CTS or CTS-to-self mechanisms solved do not exist anymore. The conclusion is that the usage of those mechanisms do not make sense in modern networks anymore. The CTS packet is not available for packet overhearing avoidance.

\section{PACKET OVERHEARING REDUCTION CONCEPT}

This concept was detailed in [4]. It is here succinctly repeated for the reader.

We propose to have the STA PHY switch to a new state, the semi-doze state, which consumes less power than the idle state but still more than the doze state.
This state is a compromise between the very low power consumption doze state and the very fast switch time from the idle state to the receive or transmit states. Each packet received is checked for destination address. If the address does not match, the PHY switches to the semidoze state for the remaining duration of the packet. The time required to detect that the packet is not relevant and the time needed to switch back to the idle state is the residual time, $\tau_{\text {res }}$. During $\tau_{\text {res }}$, the STA stays in receive state. For the remaining of the packet the STA is in semi-doze state. Only successfully received packets can have their duration and destination address identified. Therefore, only for those packets the PHY can be partially in semi-doze state.

\section{HEADER COMPRESSION CONCEPT}

The analysis done in [3] shows that the probability of undetected errors in WLAN can be high. We proposed in [3] to append to the compressed header a 16bit CRC, avoid compression when the SNR is too low, compress only data frames (not control or management frames), not to compress retransmitted frames and limit the compressed field to less than 100 bytes. These measures sharply reduce the probability of undetected errors.

We proposed in [3] to compress efficiently the WLAN header, by reducing the size of each field independently. For example, some address fields can be reduced from 6 bytes to a single byte. In total we reduce the WLAN header from 192 bits to less than 60 bits.

The compression of the layers above the WLAN MAC layer consists in comparing the previously successfully transmitted frame with the frame to be sent on the same Access Category queue. Except for the WLAN header, the headers are unknown, and therefore, the border between the last header byte end and the first payload byte is unknown and has to be evaluated by the compressor. We proposed in [3] 3 methods to evaluate this boundary. We introduced a new compression algorithm called the Fixed Asymmetric algorithm. This algorithm assumes that the difference between two consecutive frames is small and this difference consists in long streams of zeros interrupted by short bursts of ones. Therefore it compresses efficiently strings of zeros. In [3], we also defined the decompression algorithms.

\section{ANALYTICAL MODEL DEVELOPMENT}

In [4], we developed the analytical model of a standard WLAN STA power consumption. This model is based on Bianchi's analytical model of throughput developed in [5].

The analytical model was validated by comparing the results to a WLAN network simulation developed internally in Matlab language. The network simulation emulates a network where all the stations follow the Carrier Sense Multiple Access with Collision Avoidance 
(CSMA/CA) protocol, as defined in [1]. The correct behavior of the simulator itself was checked by comparing the simulator normalized throughput to the analytical models defined in [5]. Once the throughput is correct, it is guaranteed that the behavior of all the stations on the simulator access the media as defined by the CSMA/CA protocol. Each station is correctly switching PHY power states (transmit, receive and idle). The power consumption estimation for each station in the simulation is then simply accumulating the energy based on the station power state.

In this section we define the modifications of this model to support the header compression and the packet overhearing reduction methods. Additionally, we develop a single STA analytical model because the models derived from [5] are only suitable when there are at least two active STAs.

\subsection{Header compression}

An analytical model for performance analysis of a single station scenario is developed below, and existing analytical models are reused for performance analysis in the case of several stations.

\section{Sinqle Station Scenario}

The following scenario is checked: there is a single STA on the channel and it uploads a file to the AP. The data packets are compressed at the STA and decompressed at the AP. The packets are uploaded at the maximum possible data rate and the packets have a fixed payload length, $L$, in bytes. We assume no packet error and a fixed PHY rate.

Both the WLAN MAC header and the higher layer headers are compressed. The combined length of the noncompressed headers, $H$ is reduced to $H_{c}$ by compression. The lengths unit is the byte.

In order to calculate energy consumption, the packet length in bytes has to be converted to a duration. This duration depends on the PHY rate, $R_{P H Y}$ (in bits per second), as defined in

$$
\tau_{p}=\tau_{H O F D M}+\left\lceil 8 \frac{H+L}{R_{P H Y} \sigma_{O F D M}}\right\rceil \sigma_{O F D M} .
$$

$\tau_{\text {HOFDM }}$ duration is the PHY header duration in $802.11 \mathrm{a} / \mathrm{g} / \mathrm{n}$. It is defined in [1] and is fixed for a given modulation. $\sigma_{O F D M}$ is the OFDM symbol duration, which is defined in [1] to be $4 \mu s$, except for the $802.11 \mathrm{n}$ where it can be optionally reduced to $3.6 \mu \mathrm{s}$. For further analysis, the ceiling to $\sigma_{O F D M}$ will be omitted for simplification.

The duration of a compressed packet, $\tau_{c p}$, is obtained by replacing $H$ by $H_{c}$ in equation (1). Since there is only a single STA transmitting, there is no collision risk, and therefore, all frames are compressed.

The number of packets the STA needs to send to transfer its file of $F$ bytes and the time the STA spends in transmit state per second - if no compression is performed, or if compression is performed - are

$$
n_{p}=\frac{F}{L}, \quad \tau_{t x}=n_{p} \tau_{p}, \quad \tau_{c t x}=n_{p} \tau_{c p} .
$$

The time the STA spends in reception is only to receive the ACK packets and the beacons

$$
\tau_{r x}=n_{p} \tau_{A C K}+\tau_{\text {beac }} .
$$

$\tau_{\text {beac }}$ is the duration of the beacons, assuming they are not compressed.

The idle time is the average required CSMA/CA backoff time, the interval between data packet and its ACK (SIFS) and the DIFS interval, after the ACK

$$
\tau_{\text {idle }}=\left(\frac{C W_{\min }}{2}+S I F S+D I F S\right) n_{p} .
$$

The duration of the contention window $C W_{\text {min }}$ and DIFS vary with the Access Category, while SIFS is defined in [1].

The average power consumption of the STA if it does not perform compression is $P_{\text {savg }}$, as defined in

$$
P_{\text {savg }}=\tau_{r x} P_{r x}+\tau_{t x} P_{t x}+\tau_{\text {idle }} P_{\text {idle }} .
$$

If the station performs compression, the average power consumption, $P_{\text {cavg }}$, becomes

$$
P_{\text {cavg }}=\tau_{r x} P_{r x}+\tau_{c t x} P_{t x}+\tau_{\text {idle }} P_{\text {idle }} .
$$

The gain from compression, $G_{c}$, in percentage, is defined in

$$
G_{c}=\frac{P_{\text {savg }}-P_{\text {cavg }}}{P_{\text {savg }}} .
$$

It is also possible to extract the normalized throughput $S$, from this simple model. It is the proportion of time the STA transmits its payload, to the total time required to transmit this payload and accordingly, with the compressed header $S_{c}$, we have

$$
\begin{gathered}
S=\frac{n_{p} 8 L / R_{P H Y}}{\tau_{t x}+\tau_{r x}+\tau_{\text {idle }}}, \quad S_{c}=\frac{n_{p} 8 L / R_{P H Y}}{\tau_{c t x}+\tau_{r x}+\tau_{\text {idle }}} . \\
G_{t h}=\frac{S_{c}}{S}-1=\frac{\tau_{t x}-\tau_{c t x}}{\tau_{t x}+\tau_{r x}+\tau_{\text {idle }}}
\end{gathered}
$$

\section{Multiple Station Scenario}

As stated in [4], the analytical models study firstly the behavior of a single STA and then extend this to the whole network. The basic assumption is therefore, that all STAs have the same behavior. The analytical models can consequently only provide results for the case that all the STAs on the channel perform header compression.

The header that is compressed is both the WLAN MAC header and the higher layer headers. The headers duration is reduced from $H$ to $H_{c}$. Where $H$ and $H_{c}$ represent the time needed to transmit the normal header and the compressed header respectively. 


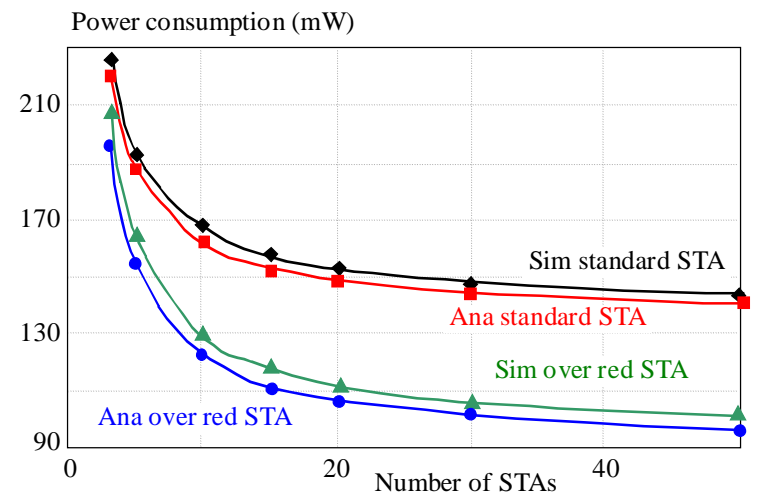

Fig. 1. Power vs $\mathrm{N}$ in saturation mode at $\mathrm{PHY}$ rate $24 \mathrm{Mbps}$

The probability of a successful transmission, $P_{s 1}$, without collision is the probability that there is a first time transmission of a given packet $\left(\tau_{1}\right)$, while no other STA transmits, under the condition that there was a transmission.

$$
P_{s 1}=\frac{N \tau_{1}(1-\tau)^{N-1}}{\tau}
$$

with $N$, the number of STAs (including the Access Point) and $\tau$, the probability of transmission. $\tau_{1}$ was obtained by Bianchi in [5] as an intermediate step:

$$
\tau_{1}=\frac{2\left(1-2 p_{c}\right)\left(1-p_{c}\right)}{\left(1-2 p_{c}\right)\left(C W_{\min }+1\right)+p_{c} C W_{\min }\left(1-\left(2 p_{c}\right)^{m}\right)}
$$

with $p_{c}$ the probability of collision.

The header duration for all packets which are transmitted first time right is $H_{c}$. As stated in Section 5 , the frames which are re-transmitted have the standard uncompressed duration $H$. The average header duration is

$$
H_{a v g}=P_{s 1} H_{c}+\left(1-P_{s 1}\right) H .
$$

$H$ is replaced by $H_{a v g}$ in all equations to obtain the average power consumption at a STA performing header compression in a channel where all STAs perform header compression.

\subsection{Packet overhearing reduction}

For the new PHY semi-doze state, the time in this state, $\tau_{\text {semi-doze }}$, is estimated in

$$
\tau_{\text {semi-doze }}=\frac{N-2}{N} \sigma_{s} \frac{L+H-\tau_{r e s}}{T_{s}},
$$

with $L$ the time needed to transfer the payload, $\sigma_{s}$, the fraction of time the STA is receiving packets successfully, and $T_{s}$, the time needed to successfully transfer a frame. Equation (16) was explained in [4].

The time in receive state for a STA that implements the partial overhearing avoidance method, $\tau_{O A r x}$, is the standard STA time in receive state subtracted by the time it is in semi-doze state: $\tau_{O A r x}=\tau_{r x}-\tau_{\text {semi-doze }}$.
Power consumption $(\mathrm{mW})$

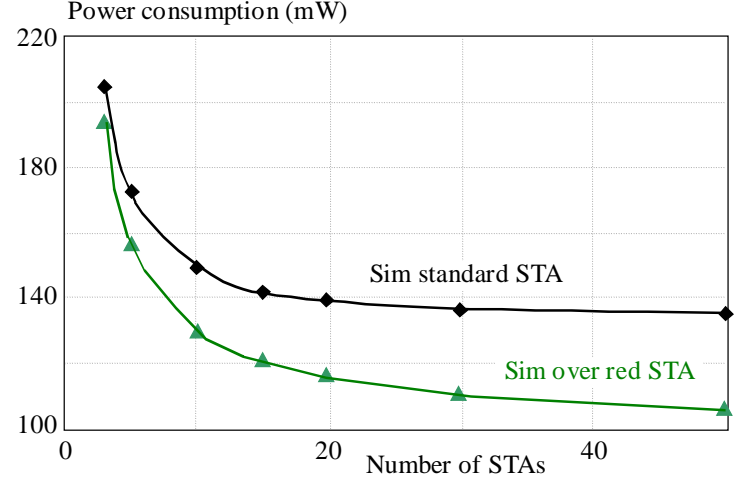

Fig. 2. Power vs $\mathrm{N}$ in non-saturation mode at $\mathrm{PHY}$ rate $24 \mathrm{Mbps}$

The resulting power consumption of a STA implementing partial overhearing avoidance is

$$
\begin{aligned}
P_{\text {OAavg }}=\tau_{\text {idle }} P_{\text {idle }}+\tau_{t x} P_{t x} & +\tau_{\text {OArx }} P_{r x} \\
& +\tau_{\text {semi-doze }} P_{\text {semi-doze }} .
\end{aligned}
$$

$\tau_{\text {idle }}$ is the time in idle state. $\tau_{t x}$ is the time in transmit state. These values were defined in [4].

\section{PACKET OVERHEARING PERFORMANCE ANALYSIS}

This section concentrates on the average power consumption parameter. Two cases are compared: a standard STA and a STA with the packet overhearing reduction method implemented. The simulator described in Section 6 was used for the simulations shown in this section. The analytical models described in [4], as well as the modified analytical model described in Section 6.2, were used in this section. For all simulations, the packet payload length is 1000 byte and the headers length is 100 byte.

The power consumption gain is depicted in Fig. 1 for a STA implementing the packet overhearing reduction method in saturation mode with a physical rate of $24 \mathrm{Mbps}$. It can be seen that with increasing number of STAs the power consumption decreases. When the STAs want to transmit they have to wait for a backoff counter timeout. With increasing number of STAs the number of collisions in the network grows resulting in higher number of retransmissions. That means the more STAs in a network the larger average value of backoff counter. When the average backoff counter value increases the STAs have to spend more time in idle state and less in the most power consuming state, $i e$ in the transmit state. If a STA integrates the packet overhearing reduction method it can stay in semi-doze state instead of receive state and thus decrease the power consumption. Another factor influencing time a STA stays in this (low power) state is an the packet duration. The lower the PHY rate the longer the packets are and the longer a STA stays in the semi-doze state thanks to the packet overhearing reduction method. In other words the power consumption gain increases as the PHY rate decreases. Figure 2 shows the power consumption when which is the channel is in non-saturation 


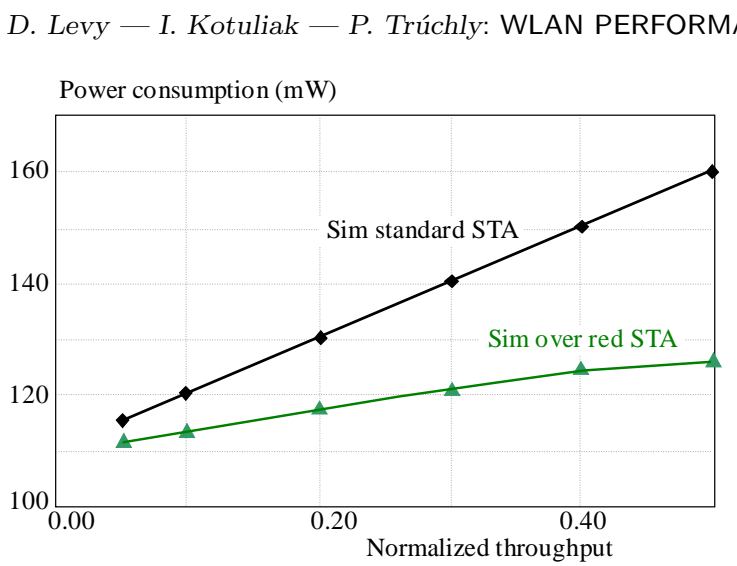

Fig. 3. Power $v s$ throughput at PHY rate $24 \mathrm{Mbps}$ with 15 STA

mode. The normalized throughput is maintained at value of 0.33 which is below saturation level for 50 STAs. With fewer STAs, the throughput is very low throughput because the STAs transmit low amount of data and they spend short amount of time in the transmit state. Therefore they also spend less time in the receive state. The same is valid for a STA implementing packet overhearing reduction method that spends less time in the semi-doze state. If a STA spends less time in the semi-doze state then it saves less power in comparison with a saturation mode. The power save gain increases as a number of STAs grows because the throughput increasis.

In Fig. 3, the case of 15 STAs at $24 \mathrm{Mbps}$ is analyzed with varying normalized throughput almost up to the saturation throughput (which is at 0.55 here). The same effect as described for Fig. 2 occurs here. Increasing the channel normalized throughput increases the proportion of time a STA is in receive state due to more retransmissions. This gives then more opportunities for a STA to be in semi-doze state.

\section{HEADER COMPRESSION PERFORMANCE ANALYSIS}

This section will describe the performance improvements by applying header compression. First, we will consider the power consumption reduction, then the throughput gain.

\subsection{Power save}

We varied the main parameters to see their effect on the STA power consumption. Unless otherwise specified the parameters that were not varied have the values specified in Table 1. All simulations were run in saturation mode using the simulator described in Section 6 , the analytical model described in [4] and the modifications given in Section 6.1.

The header reduction of 70 byte is an average result that was obtained in [3]. It is based on captured frames from real networks that were later compressed using the algorithms defined in [3].
Table 1. Default parameters choice for power save estimation when compressing the headers

\begin{tabular}{lcc}
\hline Parameter & Value & Unit \\
\hline Number of active STAs & 1 & \\
Payload & 550 & byte \\
Header reduction & 70 & byte \\
PHY rate & 6 & Mbps \\
Load & 100 & $\%$ \\
Packet generation & degenerate & \\
\multicolumn{1}{c}{ distribution } & (constant bit rate) & \\
\hline
\end{tabular}

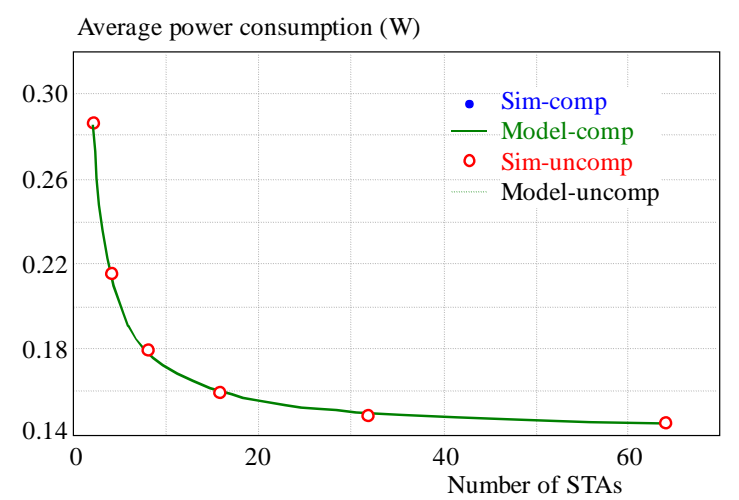

Fig. 4. Header compression power save vs number of STAs

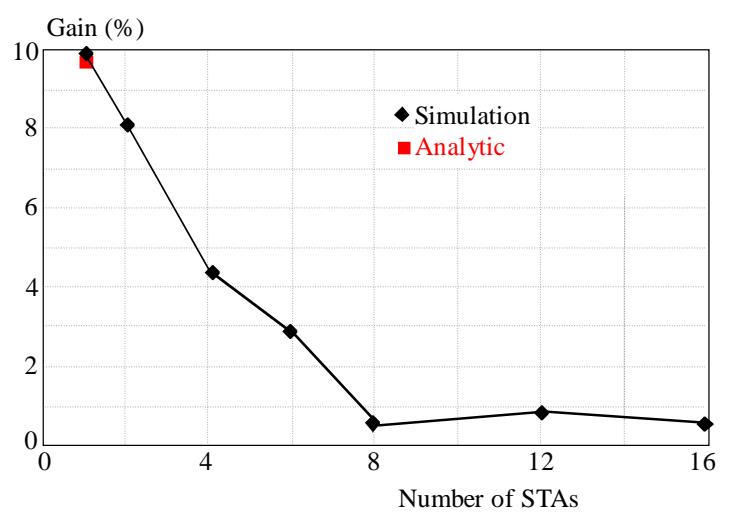

Fig. 5. File Transfer with Header compression: power save vs number of STAs

In Fig. 4 the number of STAs was varied. We see that the reduction in power consumption is negligible. The analytical model, as well as the simulation, consider an average power consumption for a saturated scenario (nonstop transfer). In this case, the header compression only achieves to squeeze more payload per unit of time, which does not reduce the average active time. In real life, the user would like to transfer a file, with a finite size. We consider the case of a Basic Service Set (BSS) where all STAs, including the AP have to transfer a file. Each file has the size of 1 MByte. In this case, it is possible to have variations of results, due to the random behavior of the backoff value before a transmission. In order to minimize this effect, each simulation was run at least 10 times and the average is shown.

We first vary the number of STAs, that transfer 1MByte file simultaneously. The result is depicted on Fig. 5. 


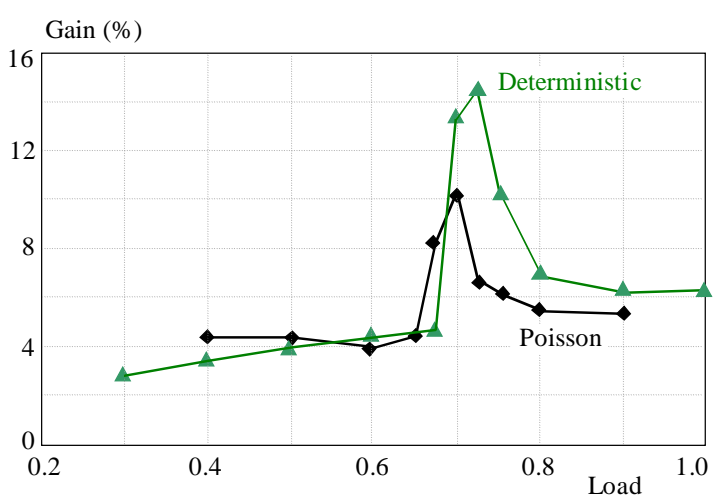

Fig. 6. File Transfer with Header compression: power saved vs load, packet generation distribution impact

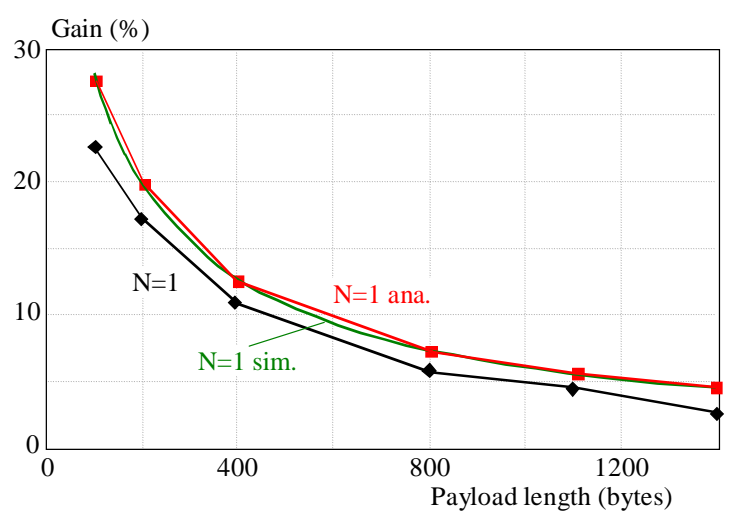

Fig. 7. File Transfer with Header compression: power saved vs payload length

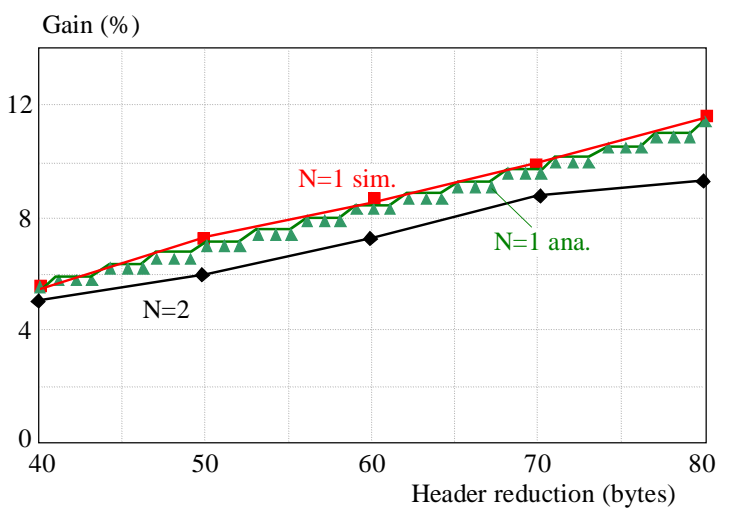

Fig. 8. File Transfer with Header compression: power saved vs header reduction amount

Results for the analytical model for a single STA was obtained as described in Section 6.1. We see a very minor underestimation of the gain by the theoretical analysis. This is due to the (uncompressed) Beacon reception time that was included in the analytical model, but not in the simulation. With increasing number of active STAs the number of collisions also increases. Increased number of collisions causes increased number of retransmissions but based on header compression algorithm the compression is only performed on the first transmission attempt therefore the ratio of compressed and uncompressed frames decreases accordingly. This behaviour results in descending value of the power consumption gain. As the number of STAs becomes high the gain slowly approaches an asymptotic value of $0 \%$.
We also investigated how the STAs load influences the power consumption gain. The results are depicted in Fig. 6 where a curve labeled deterministic represent a case with degenerate distribution (constant interval) of packet generation. The number of STAs is set to 3. Low loads represent low traffic and therefore STAs are mostly in idle state and the saved power is low. With increasing load (traffic) at certain short range the gain achieves its maximum value and for loads after this saturation the gain greatly decreases and stays almost constant. For loads above a saturation level the channel contains many collisions but backoff counter strategy ensures that some atfirst-attempt frame transmissions are successful and the header compression provides some benefit. The header compression algorithm provides the highest gain in this case around a load of 0.7 because it moves a boundary when collisions start to slightly higher value.

In order to understand the impact of the packet generation, we used the Poisson binomial distribution and compared it to the constant interval packet generation. The results are depicted in Fig. 6. Due to the packet generation interval variance with the Poisson distribution, collisions can occur at lower loads compared to the degenerate distribution. The collisions reduce the gain achieved by Header compression as the retransmitted frames are not compressed. For the same reason, the peak gain is lower in case of Poisson binomial distribution.

The effect of the payload length is depicted in Fig. 7 . As the proportion of header size to the overall frame length increases, the power saved increases. This is because the header compression reduces the size of the header, but not the size of the payload. We note the perfect match between the analytical model and the simulation for all simulated lengths. As more than a single STA transmits, collisions occur. For all the retransmitted frames, the headers are not compressed. This results in proportionally less power saved.

The effect of the header reduction amount is depicted in Fig. 8. As the frame becomes shorter, the gain increases. We see a linear behavior in the range studied: doubling the header reduction, doubles the gain in power save. As seen earlier, the existence of collision, when more than a single STA transmits, reduces the gain of header compression.

In [6] we investigated the effect of the physical rate on a general behavior of power saved and we showed decreasing gain with increasing PHY rate. The IEEE802.11 requirement of bytes mapping into OFDM symbols can explain this behavior. If a frame cannot be inserted into an integer number of OFDM symbols the last OFDM symbol is padded with insignificant bytes an average amount of which grows as PHY rate increases. Thus benefits of header compression are degraded by padding the last OFDM symbol. This problem can be nicely seen on frame payload sizes over interval from 550 to 570 bytes when padding can vary from no padding to 26 byte padding. In order to confirm this behavior, we run the analytical model for payload lengths from 540 Bytes to 600 Bytes 


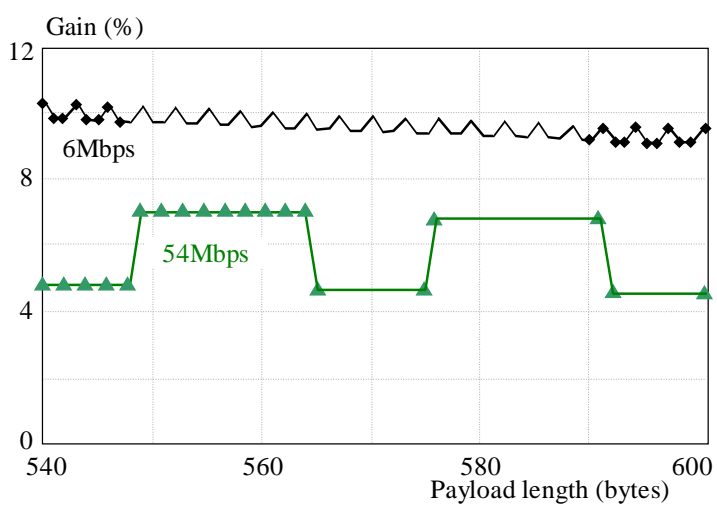

Fig. 9. File Transfer with Header compression: power saved vsPayload length and PHY rate

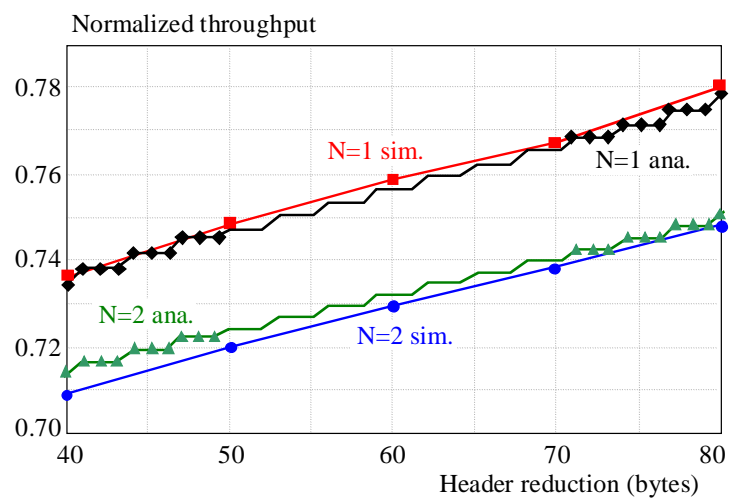

Fig. 11. Throughput gain using header compression vs header reduction

at both $6 \mathrm{Mbps}$ and $54 \mathrm{Mbps}$. The results are depicted in Fig. 9 and confirm the behavior explained above.

The small difference between the analytical model and the simulation is due to the beacon reception. In the analytical model, the constant energy consumed to receive the uncompressed beacon is taken into account, while it is not in the simulation. At higher PHY rates, it takes less time to transfer the file, therefore, the proportion of time in which the STA receives the beacon is higher. The difference between the simulation and the analytical model will then be more visible at higher PHY rates.

\subsection{Throughput gain}

The simulations run and shown in Section 8.1 also provided the normalized throughput. We show the gain on the throughput when compressing headers. For the case where a single STA transmits, we used the throughput analytical model described in Section 6.1.1. For the cases where more STAs transmit, we used the analytical model described in [5] and the modifications described in Section 6.1.2. The simulation results were obtained from the simulator described in Section 6. As was already stated, when the number of STAs increasis the probability of collisions also increasis, $i e$; the number of retransmitted frames (frames with non-compressed headers) is increasing and therefore the normalized throughput is decreasing. The highest throughput gain is achieved for only one active STA in network (gain is almost 10\%).

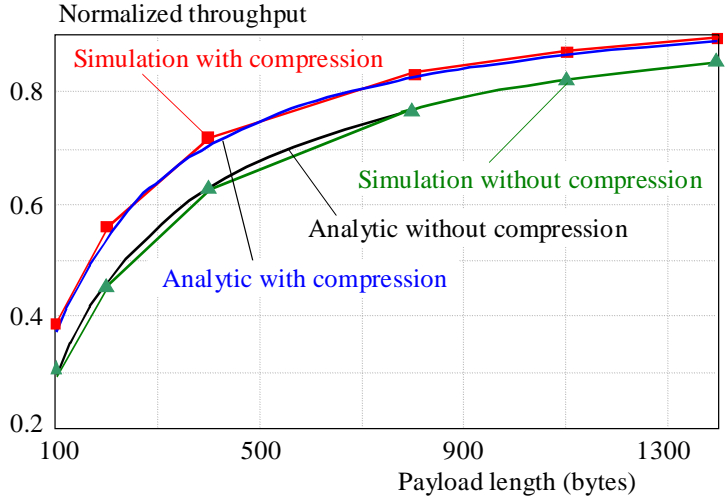

Fig. 10. Throughput gain using header compression vs payload length, with $N=1$

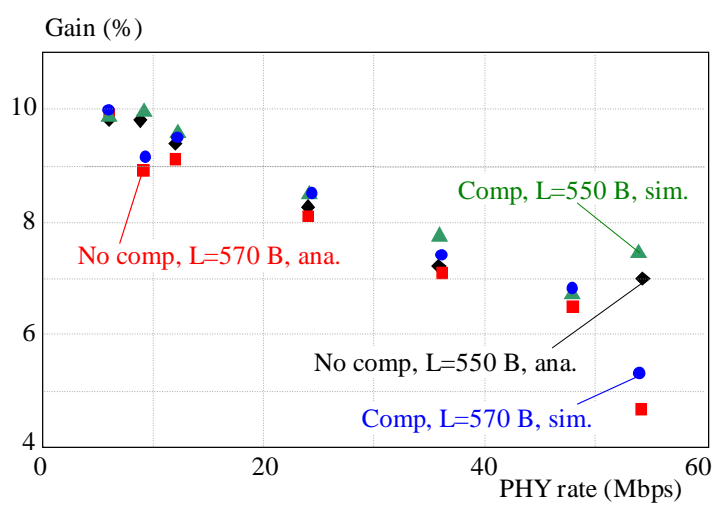

Fig. 12. Throughput gain using header compression vs $\mathrm{PHY}$ rate

How the frame length influences the normalized throughput is depicted in Fig. 10 for one active STA. In case of longer frames, the ratio of header size to payload size decreases that means the throughput grows whether or not header compression is applied. When the header compression is implemented the maximum throughput is increased as the ratio is higher. The throughput gain is almost twice higher for very short frames compared to the longest frames.

The throughput, as a function of the header reduction, is depicted in Fig. 11, for both one and two actively transmitting STAs. As explained in [3], the collisions reduce the positive impact of header compression. Therefore, for more STAs transmitting, the throughput gained from header compression is reduced.

The effect of the PHY rate is depicted in Fig. 12. We can see a general behavior of reduced gain with increasing PHY rate. The cause is similar to the one described in Section 8.1, for the power save gain: the OFDM symbol padding. On average, half of the OFDM symbol is empty, which means that part of the gain achieved by reducing the frame length (in bytes), is replaced by padding of the OFDM symbol. As the OFDM symbol contains more bytes at higher PHY rates, the gain reduction is stronger there. 


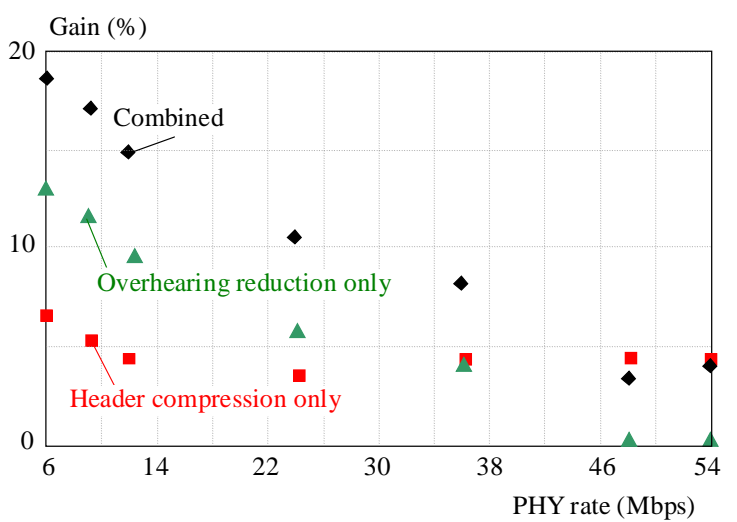

Fig. 13. Combined methods, gain vs PHY rate

\section{COMBINED PERFORMANCE ANALYSIS}

In this section, we primarily concentrate on the power consumption investigation because based on the principle of the packet overhearing reduction method, it is clear that this method does not bring a gain in data throughput. As was already mentioned in Section 8.2, the power consumption saving can be only analyzed when files with finite sizes are transmitted.

We consider the case of a BSS where all STAs, including the AP, have to transfer a file. Each file has the size of 1 MByte. In this case, it is possible to have variations of results, due to the random behavior of the backoff value before a transmission. In order to minimize this effect, each simulation was run 10 times or more, until the average change between the last run and the previous one became negligible (below 1\%). Only one STA (other than the AP) is capable of Header Compression and Packet Overhearing Reduction. Unless otherwise specified, we fix all parameters as in Table 1. The simulations were run with only the Header Compression active, and again, with only the Packet overhearing reduction active. Then with both methods activated. The simulator used is the one described in Section 6.

In order to analyse an impact of the number of STAs we can combine results depicted in Fig. 5 and Fig. 1. The former one tells us that if the header compression method is implemented with increasing number of active STAs the power consumption gain decreases. The latter one shows that if the packet overhearing reduction method is implemented the power (energy) saved increases with the number of STAs. So, both methods complement each other. It is clear that in case of one or two active STAs, no overhearing appears and gain is only from the header compression. If there is more than 8 active STAs main benefit is acquired from the overhearing reduction. For 3 active STAs, approximately the same contribution of both methods to the power saved is achieved. As seen in Fig. 7 where impact of the frame length on the power gain is depicted when the header compression is implemented, with increasing frame length the gain decreases. In case of the packet overhearing reduction the STAs remain longer in semi-doze state for longer frames so the gain is increasing. Cooperation of both methods ensures

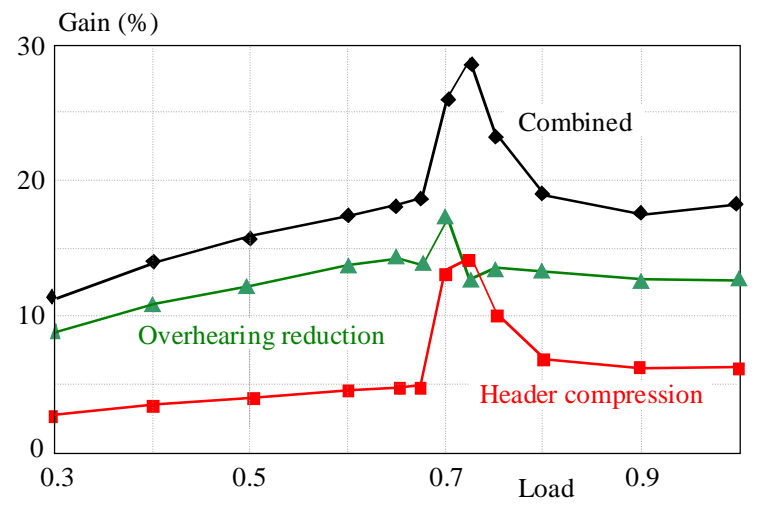

Fig. 14. Combined methods, gain vs load

almost constant gain over entire interval of the payload lengths.

The impact of the PHY rate is depicted in Fig. 13. As explained in Section 8.1, the gain slightly decreases with the PHY rate increase. This gain varies sharply at higher PHY rates and depends on the length of the frame. Looking at Fig. 1, it can be seen that the gain from Overhearing Reduction is reduced. This is simply because the duration of the same frame sent at higher $\mathrm{PHY}$ rate is shorter. Therefore, there is less overhearing time. At a certain PHY rate, the overhearing time is shorter than the residual overhearing. In this case, the gain from Overhearing Reduction completely disappears. In Fig. 13, for the frame length chosen, this is the case for PHY rate of $48 \mathrm{Mbps}$ and $54 \mathrm{Mbps}$. We can see that by combining both methods, most of the gain at low $\mathrm{PHY}$ rate comes from the Overhearing Reduction. At higher PHY rates, the gain comes only from Header Reduction.

The impact of the load is depicted in Fig. 14. Both methods have their gain reduced with reduced load. The reduced load translates to proportionally more time in PHY listen state compared to active states. Therefore, the gain achieved by header compression or packet overhearing reduction is negligible because it mainly reduces the time in PHY active states. We notice a peak of the gain at load 0.7. This load is the maximum achievable throughput for the given parameters. A further increase of the load will not lead anymore to an increase of the throughput. This means that additional load does not increase anymore the valid frames rate. The header compression compresses frames only in the first attempt. After a collision, all further attempts to transmit a given frame are not compressed headers. Packet overhearing reduction is possible because the decoded address field in the MAC header is recognized as an irrelevant address. Since the collision corrupts the frame, even the PHY header is not decoded, the packet overhearing on invalid frames is not possible.

\section{CONCLUSIONS}

In this paper we presented two methods to save power consumption in WLAN in the case of a heavily loaded 
network. The methods are header compression and packet overhearing reduction. We modified analytical models to model the effect of each of the methods. We also developed an analytical model for the case of a single active STA.

The analytical models and an internally developed simulator were used to estimate the performance improvements using the above mentioned methods. We measured up to $35 \%$ power save when combining both methods and close to 0.1 additional normalized throughput thanks to header compression.

Future work will be to implement the header compression on a real physical environment.

\section{Acknowledgements}

This work has been supported by Slovak National Research Grant Agency under project no. VEGA 1/0676/12. It is a partial result of the Research and Development Operational Program for the projects Support of Center of Excellence for Smart Technologies, Systems and Services, ITMS 26240120005 and for the projects Support of Center of Excellence for Smart Technologies, Systems and Services II, ITMS 26240120029, co-funded by ERDF.

\section{REFERENCES}

[1] IEEE Standard for Information Technology - Telecommunication and information exchange between systems - Local and metropolitan area networks - Specific requirements, Part 11: Wireless LAN Medium Access Control (MAC) and Physical Layer (PHY) Specifications, IEEE Standard 802.11-1997, IEEE Computer Society, 1997.

[2] IEEE Standard for Information Technology - Telecommunication and information exchange between systems - Local and metropolitan area networks - Specific requirements, Part 11: Wireless LAN Medium Access Control (MAC) and Physical Layer (PHY) Specifications, Amendment 5: Enhancement for Higher Throughput, Amendment to IEEE Standard 802.11-2007, IEEE Computer Society, 2009.

[3] LEVY, D.-KOTULIAK, I.: General All-Layers Combined with Efficient WLAN MAC Layer Headers Compression, In: Proceedings of International Conference on Advances in Mobile Computing \& Multimedia, 2013, pp. 123-132.

[4] LEVY, D.-KOTULIAK, I. : WLAN Power Consumption Analytical Model, In: Proceedings of Wireless and Mobile Networking Conference, 2012, pp. 101-106.

[5] BIANCHI, G.: Performance Analysis of the IEEE 802.11 Distributed Coordination Function, IEEE Journal on Selected Areas in Communications No. 3 (2000), 535-547.

[6] LEVY, D.: WLAN Power Save by Header Compression and Packet Overhearing Reduction, Informatics Sciences and Technologies Bulletin of the ACM Slovakia, extended abstracts of dissertations (2014)

[6] MCFARLAND, B. : How to Use Optional Wireless Power-Save Protocols to Dramatically Reduce Power Consumption, EETimes magazine, Design How-To (2008).

[7] BISWAS, S.-DATTA, S.: Reducing Overhearing Energy in 802.11 Networks by Low-power Interface Idling, In: Proceedings of IEEE International Conference on Performance, Computing and Communications, 2004, pp. 695-700.

[8] JACOBSON, V.: Compressing TCP/IP Headers for Low-Speed Serial Links, IETF RFC 1144 (1990).
[9] CASNER, S.-JACOBSON, V.: Compressing IP/UDP/RTP Headers for Low-Speed Serial Links, IETF RFC 2508 (1999).

10] BURMEISTER, C.-DEGERMARK, M.-FUKUSHIMA, H.HANNU, H.-JONSSON, L. E.-HAKENBERG, R.-KOREN, T.-LE, K.-LIU, Z.-MARTENSSON, A.-MIYAZAKI, A.SVANBRO, K.-WIEBKE, T.-YOSHIMURA, T.-ZHENG, H. : Robust Header Compression (ROHC): Framework and Four Profiles: RTP, UDP, ESP, and Uncompressed, IETF RFC 3095 (2001).

11] HUFFMAN, D. A.: A Method for the Construction of Minimum-Redundancy Codes, In: Proceedings of the IRE, 1952,, pp. 1098-1102.

[12] WELCH, T. A. : A Technique for High-Performance Data Compression, IEEE Computer Journal 17 No. 6 (1984), 8-19.

[13] AMIN, A.-QURESHI, H. A.-JUNAID, M.-HABIB, M. Y.-ANJUM, W. : Modified Run Length Encoding Scheme with Introduction of Bit Stuffing for Efficient Data Compression, In: Proceedings of International Conference for Internet Technology and Secured Transactions (ICITST), 2011, pp. 668-672.

14] KAZMI, H. S.-RAJA, H.: Throughput Enhancement by Cross-Layer Header Compression in WLANs, In: Proceedings of Asia-Pacific Conference on Communications, 2011, pp. 329-333.

15] CISCO SYSTEMS: Capacity, Coverage, and Deployment Considerations for IEEE $802.11 \mathrm{~g}$, white paper.

16] JU, H. J.-RUBIN, I. : The Effect of Disengaging RTS/CTS Dialogue in IEEE 802.11 MAC Protocol, In: Proceedings of International Conference on Wireless Networks, 2003, pp. 632-638.

Received 5 December 2013

David Levy was born in Sarcelles, France on March 7, 1970. Levy graduated in Electronics in 1993 with honors at Université Libre de Bruxelles, Belgium, focusing on computer networks. Levy has several patents and publications related to WLAN in particular and telecommunication in general. He worked in several startup companies as a Board and Digital Designer until 2000. He was the System Architect of the MAC layer for an ADSL modem at Alcatel and STMicroelectronics from 2000 to 2005. He was the WLAN System Architect at Texas Instruments, working on the complete WLAN transceiver in a chip for mobile phones from 2006 to 2009. Since 2010, he is a Concept Engineer for Automotive Airbag SoC at Infineon, Villach in Austria, focusing on sensor interface. From 2006 to 2009 Mr. Levy was an active member of the Wi-Fi Alliance.

Ivan Kotuliak received $\mathrm{PhD}$ degree from both Versailles University and Slovak University of Technology in 2003. From that time, he joined Slovak University of Technology and worked as researcher and assoc. prof. His research orientation is focused on network performance, including NGN architecture, wireless and mobile networking, VoIP systems and Future Internet. He has been author and co-author of more than sixty scientific papers and leads and participates on several international and national research projects.

Peter Trúchly is assistant professor at Slovak University of Technology, Faculty of Informatics and Information Technologies in Bratislava. He achieved his Master's degree in the field of informatics in 1995 and his $\mathrm{PhD}$ in applied informatics in 2004 at this university. He is interested in communications technologies, mobile applications and in the development of educational materials and e-learning platforms. His research activities cover the performance analysis of media access control and transport protocols in wireless and satellite systems. 